\title{
ManutenÇÃo Na PRÁtica de EXerCícios POR IDOSOS ${ }^{\star}$
}

\author{
Fátima A. Caromano ${ }^{\star \star}$, Maiza Ritomy Ide ${ }^{\star \star \star}$ \\ e Rachel Rodrigues Kerbauy ${ }^{\star \star \star}$
}

\section{RESUMO}

Este estudo investigou a contribuição de dois programas de treinamento físico para a manutenção na prática de exercícios físicos em idosos. Vinte idosos tiveram seu desempenho físico testado (T1) e posteriormente foram randomizados em dois grupos de 10 indivíduos. $O G_{A}$ realizou exercícios gerais. $O G_{B}$ participou de um programa de caminhada. Após 16 semanas de intervenção, todos foram reavaliados (T2). Após um ano de T2, foram novamente avaliados (T3) e também responderam uma entrevista a respeito da manutenção da prática de atividade fisica. Ambos grupos apresentaram melhora no desempenho fisico e os achados foram compativeis com as atividades referidas na entrevista. Em T3, sete dos nove participantes do $G_{A}$ mantiveram suas rotinas de atividade física. No $G_{B}$, oito dos dez o fizeram. A manutenção aumentou ou preservou o desempenho fisico, enquanto o abandono o piorou. O treinamento parece modificar o comportamento da população estudada quanto à prática de exercícios.

Palavras-chave: Exercício. Atividade fisica. Caminhada. Idoso. Manutenção. Envelhecimento.

\section{EXERCISE MAINTENANCE AMONG OLDER ADULTS}

\begin{abstract}
This study aims to investigate the effects of 2 physical training programs in the physical exercises practice maintenance in elderly persons. Twenty elderly persons had their physical performance assessed (T1). Later, subjects were randomized in two groups, with ten each. Group A performed general exercises and group B performed a program with walking exercises. After 16 weeks of intervention, subjects were assessed once again (T2). One year after T2, subjects were assessed

^ O presente estudo faz parte da tese de doutorado em Psicologia Experimental, defendida no Instituto de Psicologia da USP, pela primeira autora, orientada pela terceira autora. O trabalho não recebeu nenhuma forma de subsídio de financeiro.

$\star \star$ Professora Doutora do Curso de Fisioterapia da Universidade de São Paulo. Chefe do LaFiC Laboratório de Fisioterapia e Comportamento.

E-mail: fcaromano@uol.com.br

$\star \star \star$ Fisioterapeuta, Mestre em Ciências da Reabilitação pela Faculdade de Medicina da USP Docente do Curso de Fisioterapia da Universidade Estadual do Oeste do Paraná. E-mail: maizaide@hotmail.com

$\star \star \star \star$ Professora Titular do Curso de Psicologia da Universidade de São Paulo.
\end{abstract}


one more time (T3). In T3, subjects also answered an interview about physical activity practical maintenance. Both training interventions improved physical performance, in agree with the answers of the interview. In T3, seven of nine subjects from group A kept their exercises routines. In group B, eight of ten subjects kept the routines. Maintenance increased or preserved the physical performance, while the abandonment got it worse. Training seems to modify the population studied behavior to the exercise practice.

Keywords: Exercise. Physical activity. Walking. Aged Persons. Maintenance. Elderly.

\section{INTRODUÇÃo}

\section{O envelhecimento e a prática de atividade física}

As vantagens da prática de exercícios para idosos dependem de como se processa o envelhecimento e da rotina de exercício físico praticada. Sabe-se que os benefícios à saúde ocorrem mesmo quando a prática de atividade física é iniciada em uma fase tardia de vida, por sujeitos sedentários, sendo benéfica inclusive para portadores de doenças crônicas (ELIOT; LONG; BOONE, 1992), prevenindo principalmente as doenças associadas ao sedentarismo, como coronariopatias, diabetes, hipertensão arterial, hipercolesterolemia, acidente vascular cerebral, osteoporose, osteoartrite, e câncer de próstata, mama e cólon intestinal. Do efeito dos exercícios na prevenção e recuperação das perdas motoras decorrentes do processo de envelhecimento, conhece-se bem a relação entre treinos específicos e a melhora do órgão ou sistema exercitado, como por exemplo, prática de alongamento muscular e ganho de flexibilidade, ou treino de equilíbrio e melhora no desempenho em testes de equilíbrio (JUDGE; UNDERWOOD; GENNOSA, 1993). Para que os efeitos do treinamento de exercícios permaneçam, praticá-los deve tornar-se parte da rotina diária (MARCUS, 1995; MARCUS; RAKOWSKI; ROSSI, 1992; SHEPARD, 1993a; WILLIAMS; LORD, 1995).

Os principais motivos relacionados com o início da prática de atividade física regular, independente da idade, são o controle ou perda de peso, redução de risco de ocorrência ou recorrência de doenças, principalmente as cardiovasculares, diminuição do estresse ou depressão, melhora da auto-estima e socialização (WEINBERG; GOULD, 1996). Como principais razões para que sujeitos sedentários há anos, repentinamente, aos 70 ou 80 anos de idade, iniciem e mantenham a prática de exercícios físicos, Shephard (1990), enumera melhora da saúde, aumento da oportunidade de contatos sociais e ganhos na função cerebral. $\mathrm{O}$ autor acredita que o objetivo de melhorar a saúde seja o mais comum e que estaria relacionado com a perda de peso, diminuição do risco de doença cardiovascular e aumento na capacidade da função motora, além dos benefícios nutricionais e melhora do padrão de sono. Segundo o autor, muitas pessoas referem exercitar-se porque "isso faz com que se sintam melhor", o que se relaciona com as melhoras de saúde, o aumento na produção de beta-endorfinas e aminas biogênicas que atuam no sistema nervoso, reduzindo a ansiedade e produzindo sensação de bemestar; e as associa, ainda, com modificações positivas da auto-imagem, melhora 
na auto-estima e forte sentimento de auto-eficácia, que podem induzir a prática de outras atividades de promoção de saúde. Com a manutenção da atividade física, ocorre a diminuição nos níveis de ansiedade e depressão, influenciando na sensação de bem-estar, principalmente em pessoas com história pregressa dessa sintomatologia.

Apesar dos benefícios advindos dos exercícios físicos, existem também várias razões para justificar seu abandono. Shephard (1990) enumera como as mais freqüentemente relatadas a falta de tempo, pouca informação sobre o assunto, instalações insuficientes e cansaço. Entre os fatores negativos, aponta intensidade elevada dos exercícios e falta de qualidade do treinador.

Acredita-se que a prática de exercícios em grupo facilita sua manutenção, pelo compromisso com os demais membros do grupo, apoio social, investimento em novas relações pessoais e diversão (WEINBERG; GOULD, 1996), mas não se questiona os fatores relacionados com a manutenção da prática de atividade física, por praticantes que optaram por realizá-la sozinhos.

Shephard (1993a, 1993b) afirma que o acompanhamento através de instrução de profissionais especializados e exercícios planejados, além de testes médicos preliminares, apóiam o envolvimento de adultos idosos em programas de exercícios, enquanto o medo de queda ou lesão em programas não-supervisionados atuam como causa de abandono.

O conhecimento dos fatores envolvidos com a manutenção ou abandono da prática de exercícios é essencial para o planejamento de estratégias a serem aplicadas durante e após o período de treinamento, visando à continuidade de sua prática, fazendo-se necessários esses estudos junto a programas básicos de exercícios.

Este estudo investigou os efeitos de dois programas de treinamento na manutenção na prática de exercícios em idosos.

\section{Metodologia}

\section{Participantes}

Participaram 20 voluntários, 18 mulheres e dois homens, com média de idade $68,7 \pm 3,5$ anos. Os idosos foram selecionados segundo os critérios de não fumar, ser socialmente ativo na comunidade, não ter praticado nos últimos cinco anos exercícios físicos ou caminhadas com percursos superiores a um quilômetro mais de uma vez por semana e não apresentar disfunções de origem mioarticular, neuromotora ou cardiovascular nem doença crônica impeditiva das atividades físicas a serem treinadas.

\section{Local}


Todas as atividades foram realizadas no Laboratório Comportamento e Saúde do Instituto de Psicologia da USP e LaFiC Laboratório de Fisioterapia e Comportamento.

\section{Procedimentos}

Os sujeitos foram randomizados, através de sorteio, em dois grupos de estudo: $\mathrm{G}_{\mathrm{A}}$ (exercícios gerais) ou $\mathrm{G}_{\mathrm{B}}$ (caminhada). Após a formação de dois grupos, todos os participantes submeteram-se à avaliação do desempenho físico (T1), descrita em protocolo.

A avaliação do desempenho físico consistia de um conjunto de nove testes, realizados em dois dias consecutivos da mesma semana, durante o período da manhã. Os exames avaliaram as principais disfunções decorrentes do processo de envelhecimento: flexibilidade, força muscular em membros superiores e inferiores, postura, marcha, equilíbrio, desempenho motor manual, função cardiopulmonar e composição corporal, conforme rotinas estabelecidas na literatura.

Em seguida, os participantes dos $G_{A}$ e $G_{B}$ iniciaram os treinamentos de exercícios físicos, elaborados para este estudo, por dezesseis semanas, duas vezes por semana, em sessões de uma hora de caminhada ou exercícios físicos gerais. O programa de exercícios gerais incluiu exercícios de respiração, alongamento, postura, coordenação, força muscular e resistência cardiovascular. A freqüência cardíaca dos participantes, durante os exercícios gerais e as caminhadas, variou entre $40 \%$ e $70 \%$ da freqüência cardíaca máxima calculada. Ao final dos treinamentos, os participantes dos $G_{A}$ e $G_{B}$ submeteram-se à reavaliação de desempenho físico (T2), utilizando o mesmo protocolo de T1. Um ano após o final dos treinamentos, os participantes dos dois grupos foram contatados e convocados para nova reavaliação do desempenho físico (T3). Dos 20 participantes, 18 sujeitos compareceram à reavaliação, um faleceu e o outro mudou de país. Nessa ocasião realizou-se uma entrevista sobre a manutenção da prática de atividade física durante o ano. O participante que se encontrava fora do Brasil respondeu a entrevista por telefone.

Para determinação dos fatores ligados ao comportamento de manutenção ou abandono da prática de exercícios gerais ou caminhada, realizou-se juntamente com T3 uma entrevista, descrita no Quadro 1. As questões foram lidas aos participantes, na seqüência descrita. Se necessário, foram relidas com o objetivo de garantir sua plena compreensão (CAROMANO; KERBAUY, 1999).

\section{AnÁlise dos Dados}

Os dados referentes ao desempenho físico foram submetidos a análise estatística através da análise de variância ANOVA 1 -way, para determinar a significância das diferenças de cada variável entre os grupos. Com o mesmo objetivo, utilizouse também o teste não paramétrico de Kruskall-Wallis. Nos diferentes tempos, as variáveis foram analisadas através do teste de Wilcoxon. 
O resultado da coleta de dados das entrevistas foi analisado por leituras repetidas das respostas fornecidas pelos entrevistados. Organizou-se um roteiro para análise dos dados sobre os fatores de manutenção ou abandono da prática de exercícios indicados pelos participantes, descrito no Quadro 2 (CAROMANO; KERBAUY, 1999).

Considerou-se que houve manutenção da prática de exercícios físicos quando o participante - independente da modalidade, freqüência ou intensidade - continuara realizando-os durante o período de um ano após o término dos treinamentos. Contrariamente, considerou-se abandono da prática de exercícios físicos a interrupção total da prática de exercícios físicos. Os critérios estipulados para caracterizar a manutenção estão sintetizados na Tabela 1 (CAROMANO; KERBAUY, 1999).

\section{Resultados}

\section{Manutenção da prática de exercícios físicos após um ano}

Os participantes foram incluídos em um dos critérios descritos na Tabela 1. $\mathrm{No}_{\mathrm{A}}$, sete dos nove participantes que compareceram ao T3 relataram manutenção, contra dois que abandonaram os exercícios. Desses sete, dois realizavam as atividades treinadas, dois as realizavam parcialmente e introduziram caminhadas, dois mantiveram as atividades treinadas e associaram outra modalidade de exercícios físicos e somente um participante optou por outra modalidade. No $G_{B}$, dos dez participantes que responderam à entrevista no $\mathrm{T} 3$, dois abandonaram a prática de exercícios e oito participantes a mantiveram. Destes, três mantiveram os exercícios treinados, um participante manteve parcialmente os exercícios treinados, um manteve os exercícios treinados associado a outra modalidade e três optaram por outra modalidade de exercício físico.

\section{Análise dos efeitos da manutenção ou abandono da prática de exercitar-se no desempenho físico}

Para mensurar a prática de exercícios durante os períodos de treinamento e manutenção, criou-se o Índice de Atividade (IA), um valor numérico que exprime a quantidade da prática de exercícios em um determinado período, considerando a freqüência de realização por semana ou mês (f), a duração do treinamento (d) e a quantidade e modalidade dos exercícios realizados no período (qm). Assim, IA = (f x d x qm): 100 (CAROMANO; KERBAUY, 1999). O IA estimado para um ano foi pareado com o IA obtido durante o período de um ano após o término do treinamento, sendo este último expresso em porcentagem do primeiro. No $G_{A}$, quatro dos sete participantes que mantiveram a prática de exercícios físicos aumentaram seu índice de atividade acima de $100 \%$, enquanto três a reduziram entre $95,9 \%$ e $65,6 \%$, dos quais dois por intercorrências clínicas (CAROMANO; KERBAUY, 1999).

$\mathrm{No}_{B}$, cinco dos oito participantes mantiveram a prática de exercícios físicos com IA acima de $100 \%$ e três diminuíram este índice entre $12 \%$ e $83,3 \%$, dos 
quais um por intercorrência clínica. Considerando-se os 15 sujeitos que mantiveram a prática de atividade física, nove aumentaram o IA e somente cinco o reduziram, sendo que apenas um abaixo de $65 \%$. Verifica-se assim que, além de encontrar alta proporção de participantes mantendo a prática de exercícios, o nível de atividade para a maioria (11 de 15 participantes) manteve-se próximo de $100 \%$ ou acima, chegando a atingir 500\%. Os dados estão expressos na Tabela 2.

\section{Relação entre índice de atividade e índice de evolução (ia x iev)}

O IA mínimo, igual a zero, registrava o abandono da atividade física, não tendo sido estabelecido limite para valor máximo. Consoante com o objetivo de verificar os efeitos conjuntos do treinamento, um Índice de Evolução (IEv) foi criado para representar a evolução do desempenho de cada participante em todos os testes aplicados, sendo IEv = valor do teste 1 , variável $1+$ valor no teste 1 , variável $2+$ valor no teste $2+$.... .valor no teste $n$, variável $n$. $O$ valor de cada variável estudada foi atribuído da seguinte forma: em caso de piora, -1, em caso de manutenção, +1 e, em caso de melhora, +2 (CAROMANO; KERBAUY, 1999). O IEv variou de -19 (piora em todos os exames aplicados no T3 em relação a T2) até +38 (melhora em todos os exames aplicados no T3 em relação ao T2). A relação entre IA e IEv reflete a relação entre a prática de exercícios e a evolução do desempenho, no conjunto dos testes aplicados.

A estreita relação entre ambos os valores mostra que, quanto maior a prática de exercícios, maior a melhora na evolução, e quanto menor a prática - neste caso, o abandono -maior o número de pioras verificadas, para ambos os grupos.

\section{EVOLUÇão do DESEMPENHO FÍSICO}

Na mensuração da flexibilidade (realizada através do teste dedo-chão fotografado) observou-se que esta não foi afetada nem pela manutenção, nem pelo abandono da prática de atividade física.

A postura, analisada pela observação fotográfica da postura, em T3 demonstrou que a altura aumentou em quatro participantes e se manteve em cinco participantes do $G_{A}$, aumentou em dois e se manteve em sete dos participantes do $G_{B}$. No caso específico da postura, percebe-se que tanto a manutenção quanto o abandono afetaram a evolução da qualidade da postura, de forma positiva e negativa, respectivamente.

$\mathrm{Na}$ avaliação da marcha encontrou-se uma relação clara entre manutenção da prática de exercício e melhora, ou entre abandono e piora.

A força dos músculos dos membros inferiores estudados manteve-se ou melhorou para os praticantes de atividade física e piorou para os que a abandonaram. A força dos músculos dos membros superiores estudados não apresentou variações significativas, provavelmente porque os idosos mantiveram atividades musculares com as mãos, necessárias nas rotinas diárias.

Quanto ao equilíbrio, sua manutenção pode estar relacionada com a preservação da força muscular dos membros inferiores. 
Na avaliação do desempenho motor manual, observou-se que a manutenção da prática de exercícios físicos não é suficiente para manter a melhora alcançada com o treinamento.

No teste de caminhada de 12 minutos, verificou-se a tendência para aumentar a distância percorrida pelos participantes dos dois grupos. Associam-se estas melhoras cumulativas do treinamento e da manutenção à melhora ou manutenção da força muscular no membro inferior, qualidade da marcha, postura e equilíbrio.

A pressão inspiratória foi preservada com a manutenção dos exercícios e piorou com seu abandono; e a pressão expiratória não se alterou desde o $\mathrm{T} 1$, nos dois grupos. Como a melhora da pressão expiratória é essencial na limpeza brônquica, é fundamental a inserção de exercícios que produzam o aumento dessa força.

No estudo da composição corporal percebe-se uma tendência à diminuição do peso e da porcentagem da gordura corporal com a manutenção dos exercícios, indicando a necessidade de longo período de atividade física para alterar estes parâmetros.

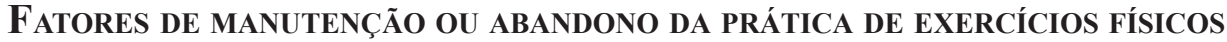

As entrevistas realizadas em T3 foram avaliadas segundo roteiro de estudo, organizando-se por assunto as informações fornecidas pelos participantes, conforme descrito no Quadro 2.

$\mathrm{O}$ primeiro tema refere-se à manutenção da prática de atividade física durante o ano que se seguiu ao final do treinamento, à capacidade de reprodução dos exercícios treinados e à introdução ou não de outra modalidade de exercícios, além dos treinados. No $\mathrm{G}_{\mathrm{A}}$, seis dos sete participantes que mantiveram o comportamento de praticar exercícios gerais demonstraram adequadamente 12 dos 12 exercícios treinados. Um participante demonstrou apenas 10 dos 12 exercícios. Dos sete participantes que deram continuidade às atividades treinadas, somente dois mantiveram as atividades tal como treinadas, enquanto quatro introduziram outras modalidades de exercício: dois sujeitos incluíram caminhadas, um acrescentou hidroginástica, um praticou também quatro exercícios que viu em uma revista e outro optou por realizar exercícios com auxílio de uma fisioterapeuta. No $\mathrm{G}_{\mathrm{B}}$, dos oito participantes que mantiveram o comportamento de caminhar, cinco continuaram a realizar a atividade como treinada, embora um apenas parcialmente. Cada um dos outros três participantes substituíram a caminhada por outra atividade: futebol amador, natação e ginástica em grupo para terceira idade.

Sobre o segundo assunto o reconhecimento da prática de exercício físico como fator de modificação e/ou manutenção de aquisições físicas alcançadas os sete participantes do $\mathrm{G}_{\mathrm{A}}$ que mantiveram o comportamento de exercitar-se expressaram o reconhecimento dessa prática como fator propício à melhora ou manutenção dos benefícios físicos alcançados. Dois participantes utilizaram a expressão "sentir-se melhor", tendo outro participante referido perceber influência dessa melhora no desempenho do trabalho. Cinco dos sete participantes 
fizeram associação com melhoras relativas a sintomas físicos. Um participante referiu ter percebido diminuição na necessidade de remédios e outro relatou alterações positivas no humor. $\mathrm{No}_{\mathrm{B}}$, todos os oitos participantes que mantiveram o comportamento de exercitarem-se associaram a prática de exercício físico com a modificação e/ou manutenção dos benefícios físicos alcançados; destes, apenas dois não especificaram os benefícios. Três participantes referiram como benefícios melhoras físicas associadas a sintomas e os dois referiram sentir-se melhor. Um participante referiu sentir-se "mais forte"; e outro associou com a melhora do sono e desempenho ao andar, enquanto a participante $6 \mathrm{~B}$ referiu melhora de atenção, apetite e sono.

Quanto à relação da prática de exercícios físicos com modificações pessoais e/ou de estilo de vida (terceiro assunto), seis dos sete participantes do $\mathrm{G}_{\mathrm{A}}$ que mantiveram a prática de exercícios referiram tais modificações, associadas a aumento na confiança e melhora no desempenho no trabalho, aumento nos cuidados pessoais, e ainda mudança no relacionamento familiar. No $G_{B}$, dos oito participantes que apresentaram manutenção do comportamento, somente três referiram mudanças pessoais ou no estilo de vida, relacionando com ganho de sociabilidade, melhora no quadro de emocional e afirmações generalizadas que "a vida ficou melhor" por causa dos exercícios. Dentre os que mantiveram comportamento de exercitar-se, a sinalização da prática de exercícios físicos como situação "indispensável" foi presente na fala de todos: os sete participantes do $G_{A}$ e os oito do $G_{B}$.

Sobre as orientações fornecidas pelo experimentador no final dos treinamentos, para introduzir adaptações na prática de exercícios físicos, verificou-se que no $G_{A}$ e $G_{B}$, doze participantes puseram em prática as orientações. Embora todos os participantes do $\mathrm{G}_{\mathrm{A}}$ e $\mathrm{G}_{\mathrm{B}}$ tenham treinado em grupos, ao final do treinamento receberam orientações que lhes permitiam praticar os exercícios sozinhos. Certamente devido à modalidade praticada, os grupos tiveram comportamentos opostos: os participantes do $\mathrm{G}_{\mathrm{A}}$ praticavam os exercícios predominantemente sozinhos e os do $\mathrm{G}_{\mathrm{B}}$ faziam suas caminhadas acompanhados. Os que praticaram exercícios em grupo referiram principalmente ganhos em sociabilidade, enquanto os que treinaram sozinhos alegaram como razões para tanto a liberdade de horário e o fato de não precisar se deslocar (para juntar-se a alguém ou instituição) para se exercitar.

Finalmente, quanto à especificação de fatores que facilitaram ou dificultaram a prática de exercícios, houve consenso sobre a percepção da melhora das condições físicas ou de suas decorrências como fator que contribui para manter a prática. Dentre os demais fatores mencionados, os que mantiveram a prática da caminhada $\left(\mathrm{G}_{\mathrm{B}}\right)$ valorizaram, mais que os do $\mathrm{G}_{\mathrm{A}}$, as oportunidades de sociabilidade que propicia. Na Tabela 3 os fatores foram ordenados dos mais aos menos citados. Chama a atenção o fato de, em se tratando de idosos, apenas três participantes terem referido intercorrências clínicas como fator que dificultou a prática de exercício.

Quanto àqueles que abandonaram a prática das atividades físicas, a participante um mudou-se do país e relatou que o clima e cultura muito diferentes difi- 
cultaram a continuidade dos exercícios; outro participante alegou "ter melhorado o suficiente" e não precisar mais de exercícios; e outros dois participantes alegaram simplesmente não gostar de realizar exercícios físicos. Sobre o reconhecimento da falta de exercício físico como fator de modificação e/ou perda das aquisições físicas alcançadas com o treinamento, dois participantes disseram sentir-se fisicamente "igual" à época em que pararam de fazer exercícios; perceberam que melhoraram no período de treinamento, mas não pioraram com o abandono da atividade física, sendo também a única que manifestou intenção de voltar a praticar exercícios, assim que se adaptar ao país para onde mudou. Os participantes que abandonaram a prática de exercícios referiram-se muito pouco aos fatores que dificultaram ou facilitariam o retorno à prática de atividade física.

\section{Discussão e CONClusões}

Nos dois grupos, o número de sujeitos que abandonaram a prática de exercícios foi igual, dois. Esta porcentagem de abandono, muito baixo, é perfeitamente compatível com as registradas na literatura. $\mathrm{No}_{\mathrm{A}}$, verificou-se a manutenção da atividade treinada total ou parcialmente, associada ou não a outra modalidade, em sete dos nove participantes reavaliados. No $\mathrm{G}_{\mathrm{B}}$, observou-se dois comportamentos: manutenção total ou parcial da atividade treinada (cinco participantes) e a substituição por outra atividade para praticar (três participantes). Assim, a adoção da prática treinada foi maior no $\mathrm{G}_{\mathrm{A}}$ e a busca por outra modalidade foi maior no $\mathrm{G}_{\mathrm{B}}$. Deste, dois participantes substituíram a caminhada por atividades em grupo e o terceiro preferiu continuar fazendo exercícios na água, com a presença de uma fisioterapeuta. Nesses casos, as mudanças de atividade parecem estar relacionadas com a necessidade de realizar a prática de exercícios com outra(s) pessoa(s), no caso grupos ou a fisioterapeuta.

De forma geral, a manutenção da prática de atividade física, independente da atividade treinada, preservou ou melhorou as funções estudadas, enquanto o abandono produziu piora dessas funções sem, no entanto, haver volta aos valores alcançados no $\mathrm{T} 1$, isto é, os participantes que abandonaram a prática de exercícios físicos não perderam totalmente os ganhos obtidos com o treinamento. Em algumas situações a manutenção ou abandono da prática de exercícios físicos não aumentou nem diminuiu os ganhos físicos alcançados com o treinamento.

Conclui-se que a manutenção da prática de exercícios físicos produziu melhora ou preservação dos ganhos obtidos com os treinamentos nos sistemas estudados, para idosos sedentários saudáveis. Lembramos que o envelhecimento leva a perda gradativa das funções, de modo que sua mera manutenção é um ganho importante a ser considerado.

Em T3, observou-se que os sujeitos do $\mathrm{G}_{\mathrm{A}}$ demonstraram adequadamente a execução dos exercícios treinados ao serem requisitados, inclusive na ordem em que foram treinados. Conclui-se que o tempo e a rotina de treinamento proposta foi suficiente para a aprendizagem, constatada pela demonstração, e, ainda, que a utilização da mesma seqüência de exercícios durante o treinamento parece ser um fator favorável à aprendizagem e manutenção. 
Independente dos grupos, todos os participantes associaram a prática de exercício com melhora e manutenção de benefícios físicos. As associações mais freqüentes referiram-se a condições físicas discriminadas, como diminuição de dor, melhora do sono, melhora de movimentos; a expressão genérica "sentir-se melhor", também bastante freqüente na fala dos participantes, sugere a percepção de melhora no bem-estar geral.

Quanto a alterações de natureza pessoal, no estilo de vida, o treinamento aplicado no $G_{A}$ parece ter sido mais efetivo na produção de modificações. Os participantes deste grupo referiram vários dos fatores positivos na manutenção do comportamento de realizar exercícios físicos enumerados na literatura, como o reconhecimento da melhora do estado físico e da saúde (EMERCY; HAUCK; BLUMENTHAL, 1992), a presença de apoio familiar (READY, 1996; WEINBERG; GOULD, 1996), conhecimentos básicos referentes aos exercícios (função e execução), propiciando a liberdade de realizá-los de acordo com as necessidades e disponibilidade de tempo (WILSON; WAGNER; DWYER, 1991), e elevação da auto-estima (WEINGBERG; GOULD, 1996).

Os dados deste trabalho são compatíveis com os de Emery, Hauck e Blumenthal (1992), que estudaram a manutenção dos efeitos de um programa de exercícios físicos gerais aplicado a 101 sujeitos (com média de $67 \pm 5$ anos). Destas, 85 foram ouvidas e 80 reportaram, em entrevistas, a continuidade das atividades treinadas. A manutenção foi associada com melhora cardiopulmonar, reações psicomotoras e diminuição da ansiedade. As razões referidas pelos participantes para a continuidade dividiram-se entre melhora no estado físico e melhora da saúde. Os autores não estudaram as razões do abandono da atividade por cinco participantes da população estudada.

Quanto às caminhadas, nossos resultados diferem parcialmente dos obtido por Ready (1996), que estudou a manutenção da realização de caminhadas em 21 mulheres com idade média de $62 \pm 4,8$ anos, portadoras de nível elevado de colesterol sérico, um ano após o término de um programa supervisionado de caminhada, com duração de seis meses e freqüência de cinco dias por semana, uma hora por dia, numa intensidade de $54 \%$ da freqüência cardíaca máxima. Encontrou diminuição significativa do colesterol total, do peso e da massa gordurosa. No entanto, a média de tempo de caminhada por semana diminuiu significativamente de quatro horas e meia para menos de três horas em 6 meses. Após 12 meses, $30 \%$ dos participantes continuaram a caminhar mais de quatro horas por semana, $50 \%$ andavam de duas a quatro horas e $20 \%$ não se preocupavam mais em realizar caminhadas. Os participantes que alcançaram maior redução nos níveis sérico de colesterol no final do treinamento mantiveram melhores tempos de caminhadas no período de um ano, reforçando a idéia de que a informação sobre a evolução clínica, nesse caso através de exames laboratoriais, influencia positivamente o comportamento dos participantes. $\mathrm{O}$ autor encontrou como principais fatores associados à manutenção da caminhada, sentir-se menos cansada ou "melhor" e o encorajamento da família e amigos. Aconselhamento médico ou fatores associados à saúde não estavam associados à manutenção do 
comportamento de realizar caminhada. Fatores externos, como variações da temperatura e lesões mioarticular, foram citadas como razões para não caminhar. Concluiu também que a insatisfação com a atividade de caminhar já ao final do programa, a perda da oportunidade de sociabilidade e a ausência de feedback como o fornecido durante o treinamento supervisionado, foram as causas do decréscimo ou abandono da atividade após o programa supervisionado. Verifica-se neste estudo que nenhum dos participantes optou por outra modalidade de atividade física, relatando mesmo não ser esta de sua preferência por falta de contatos sociais, falta de informação de um profissional, intercorrência clínica e falta de prazer em realizar a atividade treinada.

No caso do presente estudo, a orientação dada aos participantes no final do treinamento, de escolher outras modalidades ou atividades caso sentissem necessidade, parece justificar a diferença entre os resultados encontrados e os de Ready (1996). Além disso, embora a relação entre fisioterapeuta e idosos não constituísse objeto de estudo nesta pesquisa, foi possível constatar, especialmente por ocasião da entrevista, que os vínculos estabelecidos, tanto de confiança no profissional quanto às relações afetivas construídas ao longo dos quatro meses de treinamento, também influenciaram a conduta de manutenção da prática de exercícios físicos. Os participantes relataram estar satisfeitos com a forma de praticar os exercícios escolhidos.

Quanto aos comportamentos associados ao abandono da prática de exercícios físicos, as razões alegadas pelos participantes que não mantiveram os exercícios são compatíveis com as da literatura: não gostar de exercícios físicos ou já ter melhorado o suficiente (além da participante que alegou forte interrupção da rotina, com a mudança de país). Essas pessoas não associaram a parada da prática de exercícios com modificações pessoais ou no estilo de vida, nem reconhecem pioras físicas, à exceção de uma participante. Três dos quatro que abandonaram os exercícios não têm intenção de voltar a praticá-los, sugerindo que as situações que facilitariam tal retorno são externas ao seu controle, como ordem médica, presença de dor ou presença de um acompanhante. Dentre os fatores que dificultaram a prática de exercícios, relataram o desprazer como a principal.

Observa-se que a prática dos exercícios e de seus efeitos independente da modalidade - com acompanhamento cuidadoso dos treinamentos, apoio de exames clínicos, conhecimento de algumas das melhoras alcançadas, aprendizado sobre os possíveis e desejados efeitos do exercício e sua manutenção no organismo por parte dos participantes e colocação da possibilidade de mudanças de rotinas de exercícios de acordo com as necessidades, disponibilidade e preferências, parece ter influenciado na manutenção da prática de exercícios da maioria dos sujeitos.

Estes resultados, em conjunto, chamam a atenção para a relevância do treinamento na manutenção do comportamento de exercitar-se. O treinamento, organizado com a preocupação de fornecer informações e orientações básicas sobre a atividade física, ensinando a detectar melhoras no desempenho motor e buscando 
adaptar-se às necessidades e limitações dos idosos, parece ter grandes chances de modificar seu comportamento quanto à prática de exercícios.

Para tanto, é necessário preparar fisioterapeutas de modo que, ao atuarem em programas de treinamento, considerem a necessidade de intervenções visando a manutenção do comportamento aprendido pelos participantes.

\section{Quadro 1 - Entrevista realizada em T3}

\section{Quadro 2 - Roteiro para estudo das entrevistas}

Tabela 1 - Critérios de descrição da manutenção da prática de exercícios

\section{Entrevista}

1. $\mathrm{O}$ (a) senhor(a) tem continuado a realizar os exercícios que aprendeu comigo ou outro tipo de exercício físico? Sim ou não?

\section{Em caso afirmativo (manutenção),}

2a. Mostre quais exercícios vem fazendo ou descreva a caminhada ou exercícios que vem praticando.

3a. Quantas vezes por semana? Quanto tempo gasta em cada vez?

4a. $\mathrm{O}$ (a) senhor(a) faz os exercícios sozinho ou acompanhado de alguém?

5a. $\mathrm{O}$ (a) senhor(a) melhorou, piorou ou manteve-se igual, desde o término do treinamento?

6a. No que os exercícios têm lhe ajudado?

\section{Em caso negativo (abandono),}

2b. Quando parou de fazer os exercícios? Realizou outro tipo de exercício físico?

3b. Por que o(a) senhor(a) parou de fazer os exercícios?

4b. Quais as dificuldades que encontrou para continuá-los?

5b. O(a) senhor(a) melhorou, piorou ou manteve-se igual, desde que parou de fazer exercícios?

6b. $\mathrm{O}(\mathrm{a})$ senhor(a) está planejando fazer exercícios novamente?

7b. De que o(a) senhor(a) precisaria, para voltar a fazer exercícios? 


\section{Tabela 2 - Índices de Atividade (IA) dos períodos de treinamento} e de manutencão nor cuieita e nor oruno

\section{Roteiro para o estudo das entrevistas}

\section{Para os participantes que mantiveram a prática de atividade física:}

1. Reprodução dos exercícios treinados $\left(\mathrm{G}_{\mathrm{A}}\right)$ ou descrição da rotina de caminhada $\left(\mathrm{G}_{\mathrm{B}}\right)$

2. Introdução de outra modalidade de exercício.

3. Reconhecimento da prática de exercício físico como fator de modificação e/ou manutenção de benefícios físicos.

4. Relacionamento da prática de exercícios físicos com modificações pessoais e/ou de estilo de vida.

5. Referência à prática de exercícios físicos como situação indispensável.

6. Utilização das instruções fornecidas pelo experimentador no final do treinamento, para introduzir adaptações na prática de exercícios físicos.

7. Rotina de praticar os exercícios: sozinho ou acompanhado

8. Especificação de fatores que facilitam ou difícultam a prática de exercícios físicos.

\section{Para os participantes que pararam de realizar a atividade física treinada:}

1. Razões alegadas para abandono das atividades físicas.

2. Reconhecimento da ausência de prática de exercício físico como fator de modificação e/ou perda das melhoras físicas alcançadas com o treinamento.

3. Relacionamento da parada da prática de exercícios físicos com modificações pessoais e/ou de estilo de vida.

4. Referência à intenção de voltar a praticar exercícios.

5. Menção a possíveis fatores facilitadores do retorno à prática de exercícios físicos.

6. Indicação de fatores que facilitam ou dificultam a prática de exercícios físicos. 
Situação / Critério

Manteve a prática de exercícios físicos como foi treinado

Manteve a prática de exercícios físicos como foi treinado, parcialmente

Manteve a prática de exercícios físicos como treinado e associou outra modalidade de atividade física

Manteve a prática de exercícios físicos como treinado parcialmente e associou outra modalidade de atividade física

Não manteve a prática de exercícios físicos (abandono)

Optou por praticar outra modalidade de atividade física

Tabela 3 - Fatores que favoreceram ou dificultaram a manutenção da prática de exercícios, segundo os participantes que a mantiveram

\begin{tabular}{|c|c|c|c|c|}
\hline Grupos & Sujeitos & IA Treinamento & IA Manutenção & Variação \\
\hline \multirow{9}{*}{$\mathrm{G}_{\mathrm{A}}$} & 1 & 12,53 & 8,22 & $\downarrow 65,6 \%$ \\
\hline & 2 & 12,53 & - & Não foi reavaliado \\
\hline & 3 & 12,53 & 43,86 & $\uparrow 350 \%$ \\
\hline & 4 & 12,53 & 12,01 & $\downarrow 95,9 \%$ \\
\hline & 5 & 12,53 & 43,85 & $\uparrow 350 \%$ \\
\hline & 6 & 12,53 & 12,01 & $\downarrow 95,9 \%$ \\
\hline & 7 & 12,53 & 0 & Abandonou \\
\hline & 8 & 12,53 & 20,36 & $\uparrow 162,5 \%$ \\
\hline & 9 & 12,53 & 18,80 & $\uparrow 150 \%$ \\
\hline \multirow{11}{*}{$\mathrm{G}_{\mathrm{B}}$} & 10 & 12,53 & 0 & Abandonou \\
\hline & 1 & 12,53 & 27,14 & $\uparrow 216,6 \%$ \\
\hline & 2 & 12,53 & 9,40 & $\downarrow 75 \%$ \\
\hline & 3 & 12,53 & 0 & Abandonou \\
\hline & 4 & 12,53 & 14,09 & $\uparrow 112,5 \%$ \\
\hline & 5 & 12,53 & 18,79 & $\uparrow 150 \%$ \\
\hline & 6 & 12,53 & 18,79 & $\uparrow 150 \%$ \\
\hline & 7 & 12,53 & 10,44 & $\downarrow 83,3 \%$ \\
\hline & 8 & 12,53 & 62,64 & $\uparrow 500 \%$ \\
\hline & 9 & 12,53 & 1,5 & $\downarrow 12 \%$ \\
\hline & 10 & 12,53 & 0 & Abandonou, não foi reavaliado \\
\hline
\end{tabular}




\section{Tabela 4 - Fatores que dificultaram ou favoreceriam o retorno à prática de exercícios, segundo os participantes que a abandonaram}

\section{REFERÊNCIAS}

\begin{tabular}{|c|c|c|c|}
\hline \multirow{2}{*}{ Fatores } & \multicolumn{3}{|c|}{$\begin{array}{l}\text { Sujeitos que relataram o } \\
\text { fator }\end{array}$} \\
\hline & $\mathrm{G}_{\mathrm{A}}$ & $\mathrm{G}_{\mathrm{B}}$ & Total \\
\hline \multicolumn{4}{|l|}{ Favorecem/motivam (pelos benefícios que proporcionam) } \\
\hline Melhora física & 6 & 6 & 12 \\
\hline Decorrências positivas advindas da melhora física: & 8 & 3 & 11 \\
\hline $\mathrm{Na}$ auto-estima ou autoconfiança & 3 & 2 & - \\
\hline $\begin{array}{l}\text { Na disposição para o trabalho / nas relações de } \\
\text { trabalho }\end{array}$ & 3 & - & - \\
\hline Nas relações familiares & 2 & - & - \\
\hline Apoio familiar & 2 & 1 & 3 \\
\hline Oportunidade de sociabilidade ampliada & 2 & 6 & 8 \\
\hline $\begin{array}{l}\text { Oportunidade de associar atividade física com atividades de } \\
\text { trabalho ou lazer }\end{array}$ & - & 3 & 3 \\
\hline Prazer (fazer o que gosta / acha importante / faz corretamente) & 1 & 3 & 4 \\
\hline Prevenção de doenças & 4 & - & 4 \\
\hline Redução de gastos com remédios & 1 & - & 1 \\
\hline Facilitam (por aspectos relativos à própria prática do exercício) & - & - & - \\
\hline $\begin{array}{l}\text { Facilidade por independer de horário, deslocamento ou } \\
\text { companhia }\end{array}$ & 3 & - & 3 \\
\hline Confiança por ter recebido instrução profisssional & 1 & - & 1 \\
\hline \multicolumn{4}{|l|}{ Dificultam } \\
\hline Falta de companhia & 1 & 3 & 4 \\
\hline Intercorrências clínicas & 2 & 1 & 3 \\
\hline Monotonia & - & 1 & 1 \\
\hline $\begin{array}{l}\text { Falta de supervisão profissional (para assegurar correção e } \\
\text { compromisso) }\end{array}$ & - & 2 & 2 \\
\hline Tempo ruim (chuva, muito frio, calor excessivo) & - & 1 & 1 \\
\hline Custo elevado de outras modalidades & 1 & - & 1 \\
\hline
\end{tabular}

CAROMANO, F. A.; KERBAUY, R. R. Recursos para pesquisa evolutiva da manutenção da prática de exercícios físicos. Arquivos de Ciências da Saúde da Unipar, Paraná, v. 3, n. 2, p.173-178, maio/ago. 1999.

\begin{tabular}{lccc}
\hline & \multicolumn{1}{c}{ Fatores } & \multicolumn{2}{c}{$\begin{array}{c}\text { Sujeitos que relataram o } \\
\text { fator }\end{array}$} \\
\cline { 2 - 4 } & $\mathrm{G}_{\mathrm{A}}$ & $\mathrm{G}_{\mathrm{B}}$ & Total \\
$\begin{array}{l}\text { Favoreceriam /motivariam } \\
\text { "Ordem médica" para sanar algum mal físico/ dor }\end{array}$ & 2 & 1 & 3 \\
$\quad \begin{array}{l}\text { Ter companhia } \\
\text { Dificultaram }\end{array}$ & - & 1 & 1 \\
$\quad \begin{array}{l}\text { Falta de prazer } \\
\text { Falta de companhia }\end{array}$ & 2 & - & 2 \\
Falta de supervisão profissional & - & 1 & 1 \\
\hline
\end{tabular}


ELIOT, R. S.; LONG, D. R.; BOONE, J. L. Rehabilitation. In: . ELIOT, R.S. (Ed.). Stress and the heart. New York: Futura, 1992.

EMERY, C. I.; HAUCK, E. R.; BLUMENTHAL, J. A. Exercise adherence or maintenance among older adults: one year follow-up study. Psychology and Aging, Washington, v. 7, n. 3, p. 466-470, Sept. 1992.

JUDGE, J. O.; UNDERWOOD, M.; GENNOSA, A. Exercise to improve gait velocity in older persons. Arch Phys Med Rehabil, [S.L], v. 74, n. 4, p. 400-406, Apr. 1993.

MARCUS, B. H. Exercise behavior and strategies for intervention. Research Quarterly for Exercise and Sport, [S.L], v. 66, n .4, p. 319-323, Dec. 1995.

MARCUS, B. H.; RAKOWSKI, W.; ROSSI, J.S. Assessing motivational readiness and decision making for exercise. Health Psychology, USA, v. 11, n. 4, p. 257261, 1992.

READY, A. E. Walking program maintenance in women with elevated serum cholesterol. Behavioral Medicine, WASHINGTON, v. 22, n. 1, p. 23-31, 1996.

SHEPHARD, R. J. The scientific basis of exercise prescribing for the very old. Journal of the American Geriatrics Society, USA, v. 38, n.1, p. 62-70, Jan. 1990.

. Exercise and aging: extending independence in older adults. Geriatrics, Canadá, v. 48, n. 5, p. 61-64, May 1993a.

. Exercise compliance: the challenge of an aging population. Canadian Journal of Cardiology, Ontario, n. 9, supplement D, p. 72D-74D, 1993b.

WEINBERG, R.S.; GOULD, D. Fundamentos de psicología del deporte y el ejercicio fisico. Barcelona: Ariel, 1996.

WILLIAMS, P.; LORD, S. R. Predictors of adherence to a structural exercise program for older women. Psychology and Aging, Washington, v. 10, n. 4, p.617-624, Dec. 1995.

WILSON, B. R.; WAGNER, D. I.; DWYER, E. S. Adherence factors in a worksite fitness program. American Journal and Health Promotion, Cleveland, v. 5, n. 3, p.172-173, Jan./Feb. 1991.

Recebido em: Dezembro /2005

Aceito em: Junho /2006 